\title{
Experience-Dependent Regulation of the Immediate-Early Gene Arc Differs across Brain Regions
}

\author{
Michele P. Kelly ${ }^{1}$ and Sam A. Deadwyler ${ }^{2}$ \\ ${ }^{1}$ Department of Biology, University of Pennsylvania, Philadelphia, Pennsylvania 19104, and 2Department of Physiology and Pharmacology, Wake Forest \\ University School of Medicine, Winston-Salem, North Carolina 27157
}

\begin{abstract}
Previously, we demonstrated that initial acquisition of a lever-press task resulted in higher levels of activity-regulated cytoskeletonassociated protein (Arc) mRNA induction than did overtrained performance (Kelly and Deadwyler, 2002). The present study extends this finding by characterizing (1) the behavioral regulation of Arc protein expression, (2) the time course of decay of Arc mRNA signal in different brain regions immediately after the initial acquisition session, and (3) the persistence of Arc mRNA induction in those same brain regions across sessions. Rats killed after initial acquisition of a simple lever-press response demonstrated significantly elevated levels of Arc protein. Interestingly, of the brain regions that demonstrated Arc mRNA induction 30 min after the acquisition session, there was a differential rate in signal decay, with only half of the regions continuing to demonstrate elevated levels of Arc at 60 min. Similarly, the extent to which Arc mRNA induction persisted across days also varied across brain regions. An unexpected outcome was that areas such as CA1 and CA3 that showed the least persistence in Arc activation immediately after the initial acquisition session showed the greatest perseverance of induction across days of training. Finally, animals less proficient at the task expressed higher levels of Arc mRNA than animals that acquired the task more quickly. Taken together, the results show that Arc mRNA and protein were regulated in an experience-dependent manner; however, the fact that the time course of Arc mRNA expression differed across brain structures suggests a differential rate of consolidation of the newly acquired behavior across specific brain regions.
\end{abstract}

Key words: activity-regulated cytoskeleton-associated protein; operant conditioning; procedural learning; gene expression; hippocampus; cingulate cortex; caudate-putamen; amygdala

\section{Introduction}

Recent evidence suggests that activity-regulated cytoskeletonassociated protein [(Arc) Lyford et al., 1995; (Arg3.1) Link et al., 1995] may play a role in the neurobiological substrate of learning (Guzowski et al., 2000, 2001; Kelly and Deadwyler, 2002), which is proposed to involve structural modification of existing synaptic architecture and, possibly, formation of new synapses (Bailey and Kandel, 1993; Stork and Welzl, 1999; Moser, 1999; DiAntonio, 2000). Arc is an effector immediate-early gene that coprecipitates with F-actin (Lyford et al., 1995) and associates with the NMDA receptor complex of the postsynaptic density (Husi et al., 2000). Interestingly, after the induction of long-term potentiation, Arc is localized to activated dendritic domains in an NMDA receptor-dependent manner (Steward et al., 1998; Steward and Worley, 2001). Furthermore, dendritically localized translation of Arc appears to be possible, because application of brainderived neurotrophic factor to synaptosomal fractions results in increased levels of Arc protein (Yin et al., 2002). Because Arc is locally regulated, it may contribute to synaptic remodeling or

Received Jan. 13, 2003; revised May 16, 2003; accepted May 16, 2003

This research was supported by National Institute on Drug Abuse Training Grants T-32 DA07246 (M.P.K.) DA13778, DA03502, and DA00119 (S.A.D.). We acknowledge Kent Vrana and Linda Porrino for technical advice as well as Matthew Lattal and Marcelo Wood for editorial feedback.

Correspondence should be addressed to Michele P. Kelly, Department of Biology, University of Pennsylvania, 319 Leidy Labs, 3740 Hamilton Walk, Philadelphia, PA 19104.E-mail: mpkelly@bbl.med.upenn.edu.

Copyright $\odot 2003$ Society for Neuroscience $\quad 0270-6474 / 03 / 236443-09 \$ 15.00 / 0$ "tagging" of activated synapses (Bear, 1997; Frey and Morris, 1998).

Arc induction has been associated with learning new behaviors. Previously it was shown that after performance of a simple lever-press task, newly trained animals exhibited higher levels of Arc transcript relative to both pseudotrained (PT) and overtrained (OT) animals (Kelly and Deadwyler, 2002). Guzowski et al. (2001) showed similar findings in rats trained on a spatial water maze. It is not surprising that pseudotraining and overtraining resulted in increased levels of Arc mRNA, because sensory stimulation (Lyford et al., 1995; Montag-Sallaz et al., 1999; Guthrie et al., 2000) and placement in a novel environment (Gall et al., 1998) induce expression of this gene. However, the fact that our former study showed that newly trained animals demonstrated higher levels of Arc mRNA relative to both pseudotrained and overtrained animals suggests that Arc is also important for synaptic processes involved in the early stages of learning new behaviors.

This study was undertaken to determine the time course of decay in Arc levels during the hour immediately after the acquisition session as well as the persistence of Arc mRNA induction across days of training. To determine the rate of decay of taskinduced Arc expression, four groups of rats were compared: home cage controls (HC), newly trained animals killed $30 \mathrm{~min}$ (NT) or $60 \mathrm{~min}$ (NT-60) after the session, and OT animals killed $30 \mathrm{~min}$ after their eighth lever-pressing session. To examine the 
persistence of Arc mRNA induction across days of training, peak levels of expression (30 min post-session) were measured in animals receiving $1,2,3,8$, or $11 \mathrm{~d}$ of training. It was hypothesized that significantly increased Arc expression would be restricted to periods of improving behavioral performance and that, because the brain regions of interest compose different anatomical circuits, the rate at which Arc mRNA levels declined, both immediately after a session and across days of training, would differ across these various structures.

\section{Materials and Methods}

Animals. Sixty-eight male Sprague Dawley rats, 3 months of age and weighing between 300 and $350 \mathrm{gm}$, were water-deprived but allowed ad libitum food for maintenance of $90-95 \%$ of their normal body weight throughout training. Animals were housed in standard conditions approved by the Association for the Assessment and Accreditation of Laboratory Animal Care, with a $12 \mathrm{hr}$ light/dark cycle. All behavioral testing occurred during the light phase of the cycle and was performed in accordance with the $\mathrm{Na}$ tional Institutes of Health Guide for the Care and Use of Laboratory Animals.

Apparatus. The testing chamber, described previously in Kelly and Deadwyler (2002), consisted of a $43 \times 43 \times 53 \mathrm{~cm}$ Plexiglas enclosure with two wall-mounted retractable levers. A water trough was positioned on the wall between the two levers. The chamber was illuminated by an overhead light and enclosed inside a commercial sound attenuated cubicle (Industrial Acoustics, Bronx, NY).

Behavioral paradigm. Rats were handled and weighed daily for $\sim 2$ weeks before initiation of training. On training days, rats were trained and then given the remainder of their water ration to maintain their constant weight. Rats were trained in the same chamber each day. Rats were initially habituated to the chambers for $2 \mathrm{~d}$ and allowed to explore for $30 \mathrm{~min}$ with the lights on and levers extended. PT animals were presented with water rewards using an automated procedure that played back the time-stamped pattern of rewards generated by a trained animal that performed the lever-press task for $2 \mathrm{~d}$ (TWO-day group). Responses on the levers had no effect for the PT group. As described previously (Kelly and Deadwyler, 2002), actively trained (AT) animals were progressively shaped across $3 \mathrm{~d}$ to acquire the lever-press response. The experimenter delivered a water reward to the subject for approaching the trough (first day), then for approaching the levers (second day), and then for pawing and ultimately pressing the levers (third day). All AT animals acquired independent lever-pressing behavior on the third day of shaping and reached a criterion of 80 lever-press responses in this first leverpressing session (session 1). All animals trained beyond session 1 performed the lever-press task without further experimenter intervention (shaping) and completed 500 responses per session during sessions 2-11. As a result, session durations were similar for NT (killed after session 1) and OT (killed after session 8 or 11) animals.

Because previous results showed heightened induction of Arc mRNA 30 min post-session (Kelly and Deadwyler, 2002), this and a 60 min time point were chosen to characterize the decay of Arc mRNA and protein expression in experiment 1 . NT animals were killed either $30 \min (n=6)$ or $60 \min (\mathrm{NT}-60, n=6)$ after session 1 , and OT animals $(n=6)$ were killed $30 \mathrm{~min}$ after session 8 . HC animals were killed at the same time as trained animals. An OT group was used as the behavioral control group because these animals actually performed the lever-press response, and our previous study showed no significant differences between PT and OT animals with regard to Arc expression (Kelly and Deadwyler, 2002).

For experiment 2 , animals that were PT $(n=12)$, NT $(n=8)$, or performed the lever-press task for $2 \mathrm{~d}(n=8), 3 \mathrm{~d}(n=8), 8 \mathrm{~d}(n=8)$, or $11 \mathrm{~d}(n=8)$ were all killed $30 \mathrm{~min}$ after their final session. Animals were trained in three separate squads, each including a PT group for purposes of normalization and comparison. Tissue from each squad was processed in parallel, and autoradiographic data were normalized as a percentage of the PT group's mean ${ }^{35} \mathrm{~S}$ counts per minute (cpm) to account for variability attributable to differences in day of kill or efficiency of probe incorporation during in situ hybridization. PT animals were chosen for normalization purposes because previous experiments (Kelly and Deadwyler, 2002) showed that labeling in HC animals was not above background in all areas. Analyses of autoradiographic data before normalization within each squad revealed no substantial differences in labeling between PT and OT animals, as reported previously (Kelly and Deadwyler, 2002).

Tissue processing. Animals were killed via carbon dioxide inhalation. In experiment 1 , brains were freshly harvested, and one hemisphere was immediately dissected for processing by Western blot (see below). The remaining hemisphere, along with brains from experiment 2, was processed by in situ hybridization for Arc mRNA, as described previously (Kelly and Deadwyler, 2002). Slides were subsequently exposed to Kodak Biomax MR film (Kodak, Rochester, NY) for $5 \mathrm{~d}$ and then processed by NT-2 emulsion dipping (Kodak) and Nissl -staining. Autoradiographic 
images, corrected for local background, were quantified as optical densities per square millimeter using ${ }^{14} \mathrm{C}$ standards calibrated for ${ }^{35} \mathrm{~S} \mathrm{cpm}$ using ${ }^{35} \mathrm{~S}$ brain paste incorporation standards (lower limit of measurable detection $=63.93 \mathrm{cpm}$ ). No signal was present in sense-labeled sections, which verified the specificity of the probe. Measurements were taken in regions of interest (ROIs) outlined in Figure 1 and are similar to those described previously (Kelly and Deadwyler, 2002).

Arc protein was assessed by Western blot, as described by Freeman et al. (2001). After dissection, tissue samples were immediately placed in microcentrifuge tubes on dry ice. Tissue from frontal cortex that included anterior cingulate, infra/prelimbic, and piriform cortices (Kelly and Deadwyler, 2002) was first sonicated in Wang buffer [ 25 mM HEPES, $\mathrm{pH}=7.5,250 \mathrm{~mm}$ sucrose, $100 \mu \mathrm{m}$ PMSF, $1 \mathrm{~mm}$ 1,4-dithiothreitol, 10 $\mu \mathrm{m} \mathrm{Fe}\left(\mathrm{NH}_{4}\right)_{2}\left(\mathrm{SO}_{4}\right)_{2}, 0.2 \%$ Triton X-100], and the total protein concentration for each sample was determined via the bicinchoninic acid assay (Smith et al., 1985) (Pierce Chemical, Rockford, IL). Sixty micrograms of protein from a single animal were loaded per well (one well per animal), because this was determined to be within the linear range of the assay. Protein samples (six animals per behavioral condition) were subjected to denaturing SDS-PAGE (Laemmli, 1970) and then transferred (using TE70; Amersham Biosciences, Piscataway, NJ) from the gel to a polyvinylidene fluoride membrane (Immobilon P; Millipore, Bedford, MA), which was subsequently blocked in $0.5 \%$ nonfat milk/0.05\% Tween 20 .

A goat-polyclonal Arc antibody (1:100 in $1 \times$ PBS/0.5\% nonfat milk; Santa Cruz Biotechnology, Santa Cruz, CA) with apparent banding at $\sim 55 \mathrm{kDa}$ was used. Antibody binding was visualized via Biomax MR film using a mouse anti-goat HRP-conjugated secondary antibody (1:2000 in $1 \times$ PBS/0.5\% milk/0.05\% Tween 20; Santa Cruz Biotechnology) and a chemiluminescence reaction (West Pico Signal, Pierce Chemical). To control for loading and transfer, select membranes were subsequently stripped using Restore stripping buffer (Pierce), reblocked, and probed with a mouse monoclonal antibody to $\beta$-actin $(\sim 42 \mathrm{kDa} ; 1: 50,000$ in $1 \times$ $\mathrm{PBS} / 0.5 \%$ nonfat milk/0.01\% Tween 20; Abcam, Cambridge, UK), which was visualized using a rabbit anti-mouse HRP-conjugated secondary antibody (1:5000 in $1 \times \mathrm{PBS} / 0.5 \%$ nonfat milk/0.01\% Tween 20 ; Santa Cruz Biotechnology; data not shown). Optical densities per square millimeter were quantified using TINA software (Fuji Medical Systems, Stanford, CT).

Statistical analysis. Behavioral measures included session length, number of experimenter-delivered rewards, and number of presses completed, along with the timestamp of each press or delivered reward within the session. From these timestamps, the number of presses completed during and after the shaping phase of session 1 could be determined. Lever-pressing rates (LPRs) were calculated as average presses per minute for each session. "Independent" LPRs (used in correlational analyses of NT animals) specifically referred to the average presses per minute completed during session 1 , after the experimenter had completed shaping the animal. Analyses of behavioral data were performed using an ANOVA.

For experiment 1 , data for each Western blot were analyzed independently. The blot comparing HC versus NT versus OT was analyzed by an ANOVA. For analysis of the blot comparing HC versus NT-60 animals, a rank sums test was used because of the smaller sample size in the HC group that resulted from the exclusion of two aberrant lanes that were $>2$ SDs below the mean. For analyses of in situ data from experiment 1, autoradiographic data from each section were compared between groups by an ANOVA (for sections 1 and 3-4, with $\leq 2$ ROIs) or a two-way repeated measures (RM) ANOVA (for section 2, with $\geq 3$ ROIs). Post hoc comparisons used the Student-Newman-Keuls range statistics. For experiment 2, all data were normalized as a percentage of the squad's PT group mean; thus, autoradiographic data from all regions across all four sections were included in a two-way RM ANOVA. Pearson productmoment correlations $(r)$ between levels of Arc mRNA and behavioral measures of individual animals were performed on data combined across both experiments. To account for differences attributable to day of kill or efficiency of probe incorporation during hybridization, autoradiographic data for each region were normalized as reported previously (Kelly and Deadwyler, 2002).
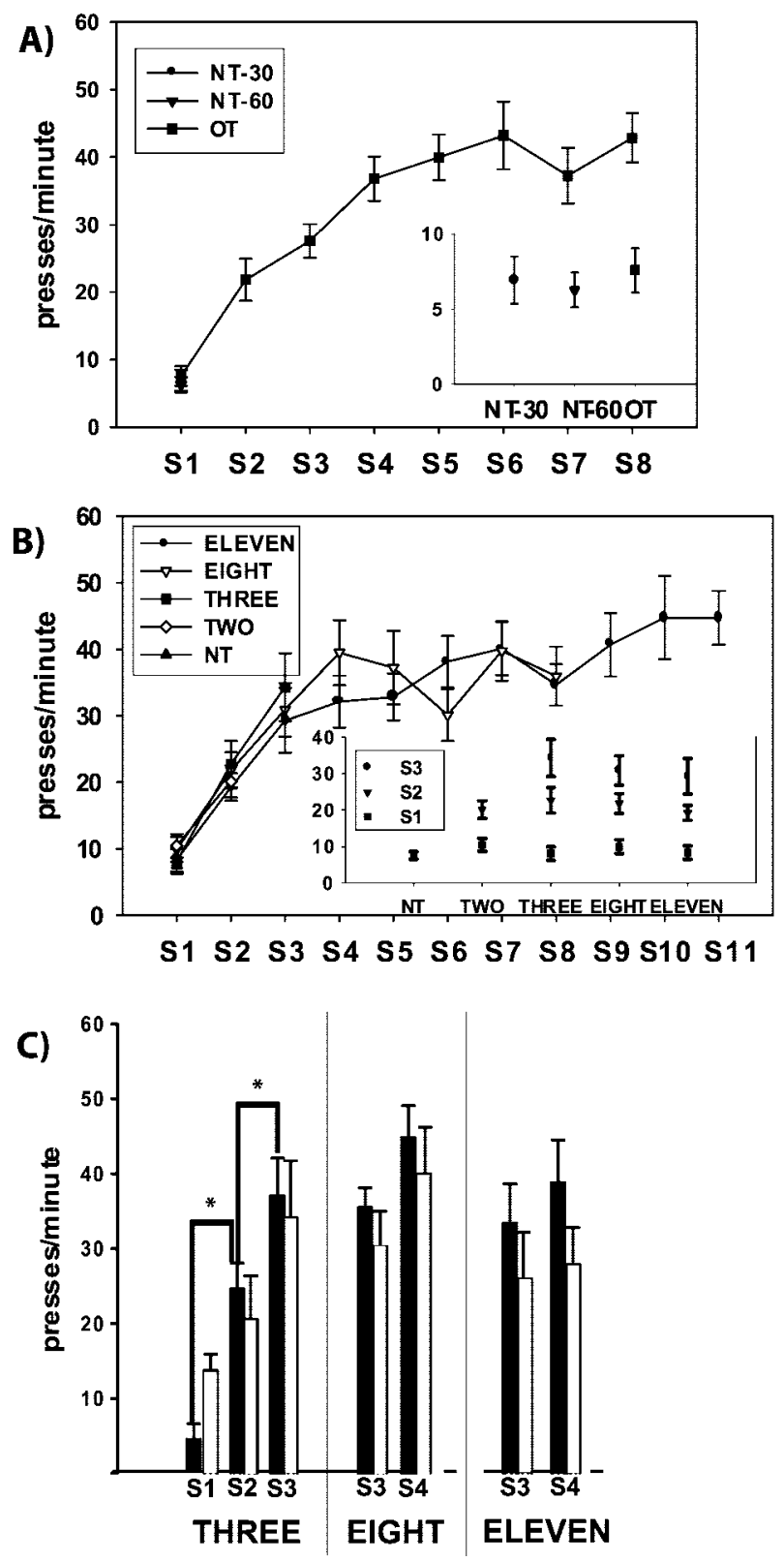

Figure 2. Behavioral data from experiments 1 and 2 . $A$, Lever-pressing rates from newly trained animals killed $30 \mathrm{~min}(\mathrm{NT} ; n=6)$ or $60 \mathrm{~min}(\mathrm{NT}-60 ; n=6)$ after their acquisition session (S1), as well as overtrained animals (OT; $n=6$ ) killed 30 min after their eighth session (S8). Because of overlapping data points, the inset at bottom right shows data for $\$ 1$ expanded. Data expressed as mean presses per minute \pm SEM. $B$, Lever-pressing rates from NT animals and animals trained for TWO, THREE, EIGHT, or ELEVEN days ( $n=8$ for each). Because of overlapping data points, the inset at bottom right shows data for \$1-3 expanded. Data are expressed as mean presses per minute \pm SEM. S1-11, Lever-pressing sessions 1-11.C, Mean LPRs ( \pm SEM) completed within the first (black bars) and last (white bars) 4 min of sessions 1-4. Post hoc analysis by Student-Newman-Keuls: significant difference, ${ }^{*} p<0.05-005$.

\section{Results}

Behavioral assessment of trained animals

In experiment 1 , to verify that OT animals had reached asymptotic performance levels before kill, LPRs across days of training were examined. Figure $2 A$ shows that LPRs of OT animals $(n=6)$ improved across days of training $\left(F_{(5,35)}=28.045 ; p<0.001\right)$. Post hoc analyses showed that animals reached asymptote on session 4 (session 4 vs $8 ; q_{(4,35)}=2.54$, NS). In experiment 2 (Fig. $2 B)$, the EIGHT-day $(n=8)$ and ELEVEN-day groups $(n=8)$ 
demonstrated similar improvement across days of training $\left(F_{(7,49)}=15.51, p<0.001\right.$; $F_{(7,49)}=15.51, p<0.001$, respectively), with no change in LPRs over the final $6 \mathrm{~d}$ of training (EIGHT, day 3 vs $8 ; q_{(2,49)}=0.18$, NS; ELEVEN, day 6 vs $\left.11 ; q_{(4,49)}=0.29, \mathrm{NS}\right)$.

Comparison of LPRs for the first and last 4 min epochs of sessions 1-3 showed improvement across all days within the THREE-day group (Fig. $2 C)\left(F_{(7,35)}=12.86\right.$; $p<0.0001)$. Post hoc analysis, however, revealed that this improvement did not occur within a session $\left(1, q_{(2,35)}=2.72\right.$, NS; 2 , $q_{(2,35)}=1.18$, NS; $3, q_{(2,35)}=0.86$, NS) but occurred spontaneously between session 1 versus $2\left(q_{(4,35)}=5.89 ; p<0.005\right)$ and session 2 versus $3\left(q_{(3,35)}=3.61 ; p<0.05\right)$. No spontaneous improvement was noted between session 3 versus 4 (Fig. 2c) (EIGHTday group, $q_{(3,35)}=2.73$, NS; ELEVEN-day group, $q_{(2,35)}=2.12$, NS).

\section{Experiment 1: decay of Arc mRNA levels} in NT animals differs across

\section{brain structures}

The inset of Figure 3 plots the overall mean $( \pm$ SEM $){ }^{35} \mathrm{~S}$ cpm across ROIs in section 2 for HC, NT, OT, and NT-60 animals $(n=$ 5 for each) from experiment 1 . Comparison of autoradiographic data showed significant induction of Arc mRNA in the NT group (Fig. 3, inset) $\left(F_{(3,112)}=22.30 ; p<\right.$ $0.001)$ relative to all other groups, whereas OT and NT-60 animals demonstrated significantly higher levels relative to $\mathrm{HC}$ controls (Fig. 3A). As demonstrated previously (Kelly and Deadwyler, 2002), Arc mRNA was expressed at different levels across these ROIs (Fig. $3 A)\left(F_{(7,112)}=\right.$ $150.21 ; p<0.001)$. In NT-60 animals, Arc mRNA levels demonstrated elevation in some [infra/prelimbic cortex (LIM); somatosensory cortex (SOM); subiculum (SUB); and caudate-putamen $(\mathrm{CPu})]$ but not all ROIs contained in section 2 (Fig. $3 A)\left(F_{(21,112)}=13.12 ; p<0.001\right)$.

The NT group also demonstrated elevated Arc mRNA levels in ROIs located in sections 1, 3, and 4 (Fig. $3 B)$. In anterior cingulate and piriform cortex, NT animals demonstrated the highest levels of Arc mRNA relative to all other groups, whereas OT and NT-60 animals differed only from the HC group (Fig. 3B) $\left(F_{(3,16)}=9.98, p<0.001\right.$ and $F_{(3,16)}=20.90$, $p<0.001$, respectively). NT animals exhibited significant induction of Arc mRNA relative to the HC group in insular cortex $\left(F_{(3,16)}=4.19 ; p<0.05\right)$ and relative to all other groups $\left(F_{(3,16)}=\right.$ $8.71 ; p<0.001)$ in the central nucleus of the amygdala (CeA) (Fig. 3B). There were no differences among HC, OT, or NT-60 animals in either insular cortex or CeA.

Figure 4 shows photomicrographs of emulsion-dipped slides (one region per section) detailing the subcellular localization of Arc mRNA in HC, NT, OT, and NT-60 animals. As described above, NT animals demonstrated more intense labeling than $\mathrm{HC}$, OT, and NT-60 animals in most ROIs. Labeling of Arc mRNA
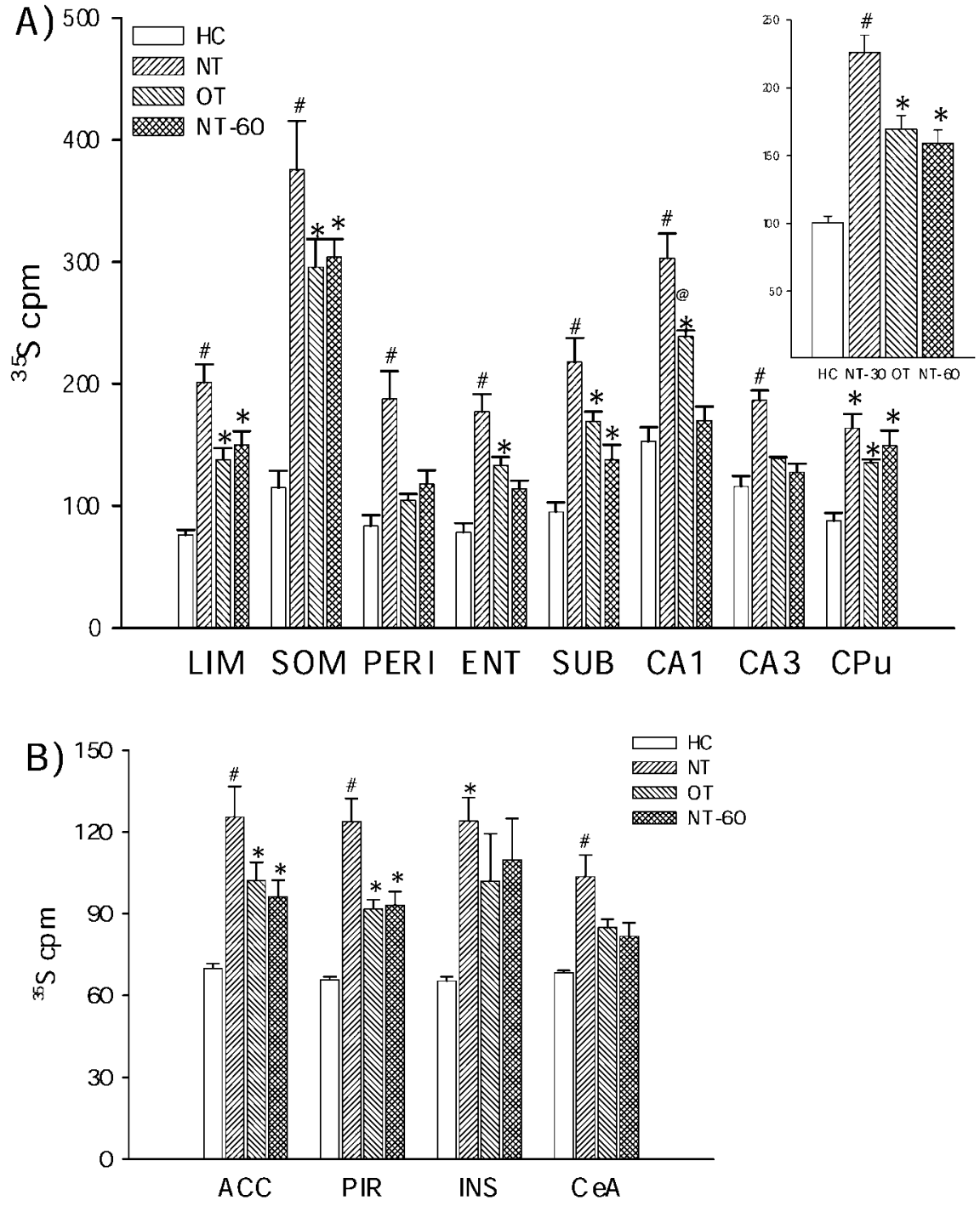

Figure 3. Arc mRNA levels return to baseline at different rates across regions. $A$, Post hoc analysis of autoradiographic data for regions of interest in section 2 (outlined in Fig. $1 B$ ) of home-caged controls ( $\mathrm{HC} ; n=5$ ), newly trained animals killed 30 min (NT; $n=5$ ) or $60 \mathrm{~min}(\mathrm{NT}-60 ; n=5)$ after the session, and overtrained animals (OT) killed 30 min after their eighth session (0T; $n=$ . The inset at top right shows overall group mean ${ }^{35} S \mathrm{cpm} \pm S E M$. $B$, Additional regions of interest from sections 1 and 3-4 outlined in Fig. $1 A,(-D)$. Data were plotted as mean ${ }^{35} \mathrm{~S} \mathrm{cpm} \pm$ SEM. LIM, Infra/prelimbic cortex; SOM, somatosensory cortex , perirhinal cortex; ENT, entorhinal cortex; SUB, subiculum; CPu, caudate-putamen; ACC, anterior cingulate cortex; PIR, piriform cortex; INS, insular cortex; CeA, central nucleus of the amygdala. Post hoc analysis by Student-Newman-Keuls: significant increase, ${ }^{\#}$ versus HC, 0 T, and NT-60; ${ }^{*}$ versus HC, ${ }^{@}$ versus NT-60, $p<0.05-p<0.001$.

was found over the Nissl-stained cell bodies, and distinct linear deposits of granules could be seen to extend from the cell body region, presumably following the course of Arc mRNA migrating into dendrites (Lyford et al., 1995; Steward et al., 1998).

\section{Only NT animals demonstrate an increase in Arc protein after} the session

Optical densities from Western blot films (Fig. 5) indicated that Arc protein in frontal cortex was significantly increased (Fig. $5 \mathrm{~A}$ ) $\left(F_{(2,14)}=4.49 ; p<0.05\right)$ in NT animals $(n=6)$ relative to both HC $(n=5)$ and OT animals $(n=6)$. The elevation in NT Arc protein levels persisted $60 \mathrm{~min}$ after the session (Fig. $5 B)\left(t_{(8)}=\right.$ $12.00 ; p<0.05)$, as indicated by a comparison of the NT-60 $(n=$ $6)$ and HC groups $(n=4)$. These results are consistent with the above-noted elevations in NT Arc mRNA levels in frontal areas 

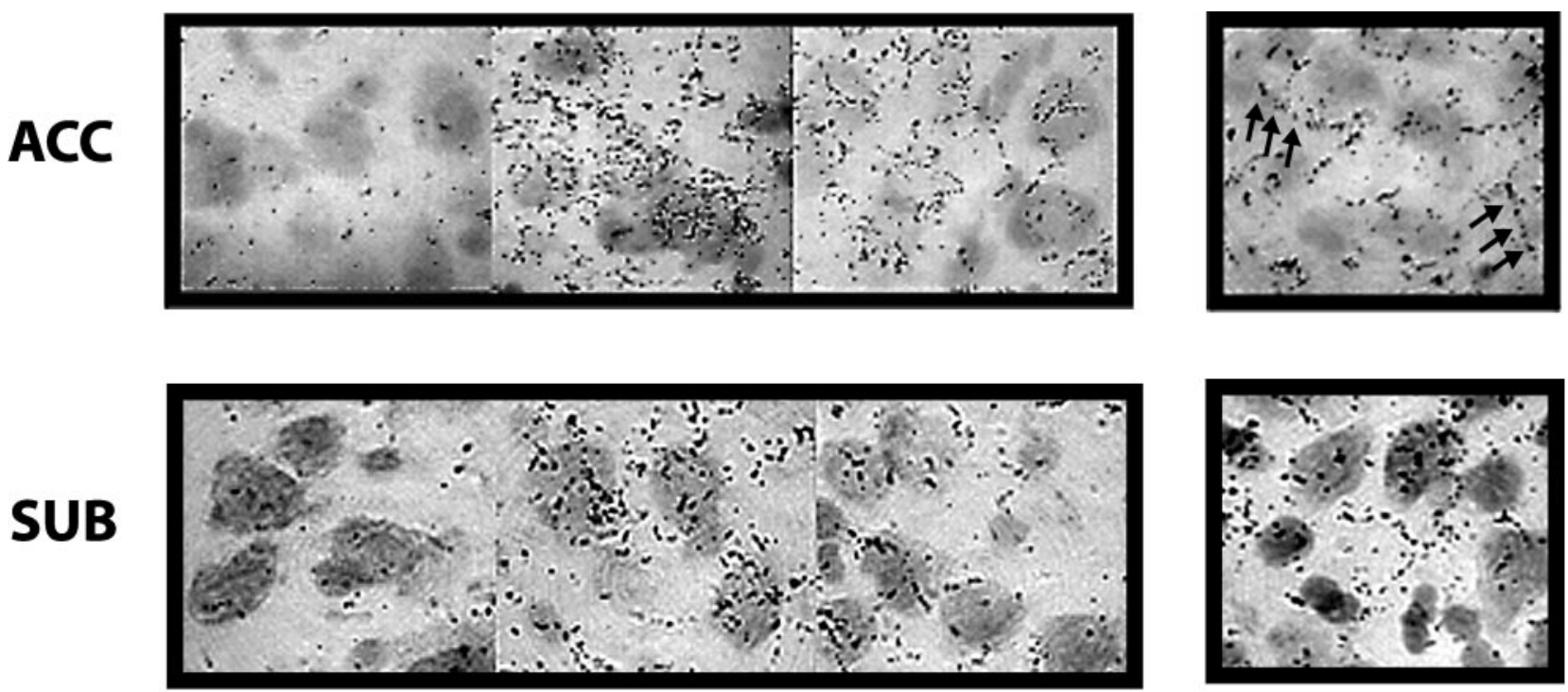

SUB
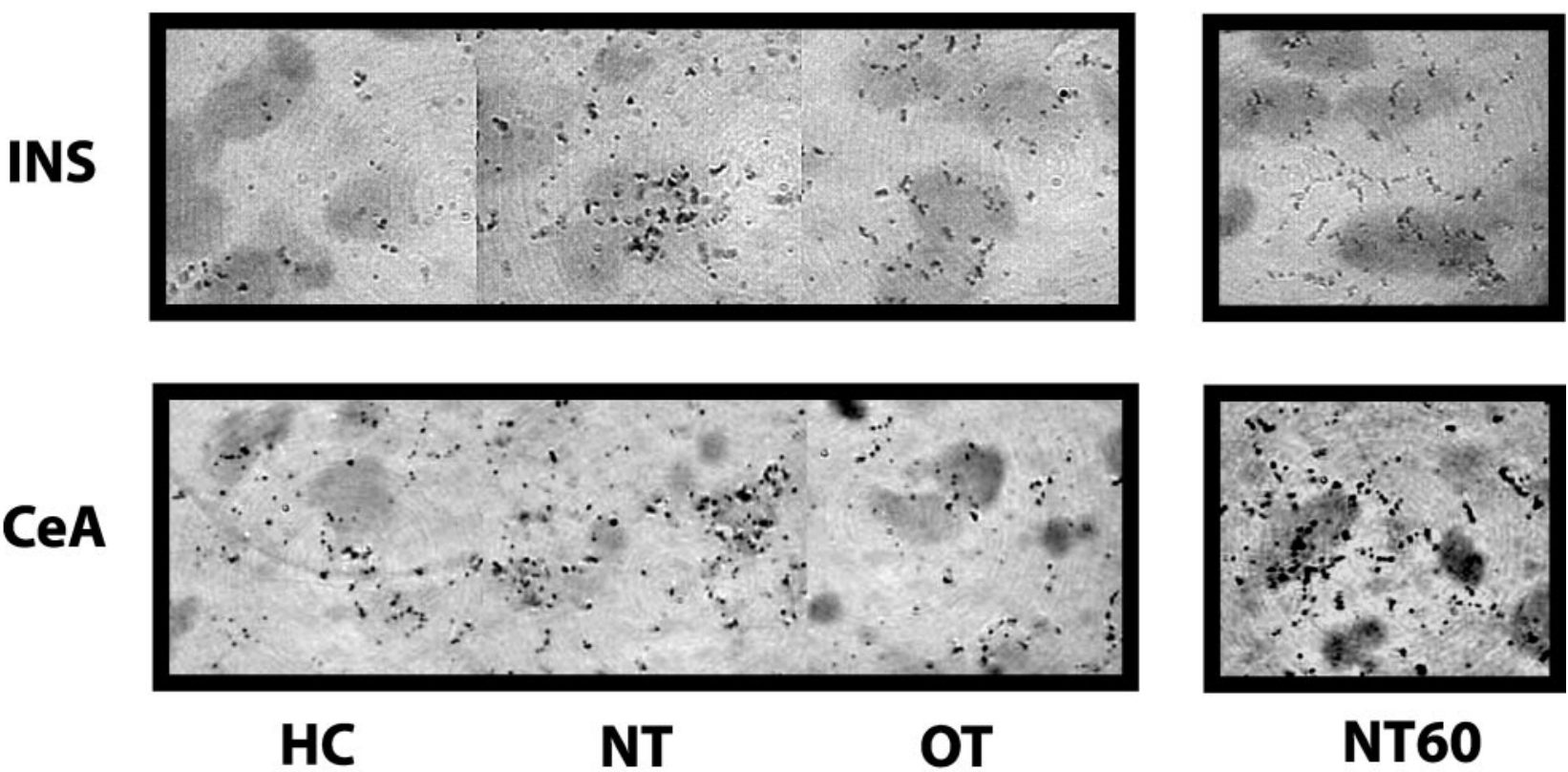

NT60

Figure 4. Somatic and matrix-associated label of Arc mRNA. Photomicrographs of slides hybridized with ${ }^{35} \mathrm{~S}$-labeled Arc antisense probes and processed by emulsion and Niss 1 stain showing label (grains) in newly trained animals killed $30 \mathrm{~min}$ (NT) or $60 \mathrm{~min}$ (NT-60) after the session, an overtrained animal (OT) killed $30 \mathrm{~min}$ after the session, and a home-caged control (HC). Note linear trails of granules extending away from cell bodies presumably labeling dendritically migrating Arc mRNA. Brightness and contrast of images were heightened for clarity and definition. ACC, Anterior cingulate cortex; SUB, subiculum; INS, insular cortex; CeA, central nucleus of the amygdala.

anterior cingulate cortex (ACC), LIM, and piriform cortex (PIR) measured 30 and $60 \mathrm{~min}$ after the session.

Experiment 2: levels of Arc mRNA induction decrease rapidly after additional training

Analyses revealed that levels of Arc mRNA in the NT and TWOday groups were higher (Fig. 6, inset) $\left(F_{(4,385)}=18.02 ; p<0.001\right)$ relative to animals in the THREE-, EIGHT-, or ELEVEN-day training groups. Additionally, the magnitude of Arc induction differed significantly across various brain regions (Fig. 6) $\left(F_{(11,385)}=9.32 ; p<0.001\right)$. Furthermore, the day on which Arc induction failed to differ significantly from overtrained levels was not the same across different brain regions $\left(\mathrm{F}_{(44,385)}=3.07 ; p<\right.$ $0.001)$. Figure 7 provides representative ${ }^{35}$ S-labeled Arc autora- diographs of horizontal sections from PT, NT, and EIGHT-day animals, illustrating the most intense labeling in NT animals.

In general, regions that showed the greatest magnitude of induction on the day of task acquisition, for example, entorhinal (ENT) and CA1, demonstrated the most persistent Arc activation across days (Fig. 6). Interestingly, these were the same regions that tended to exhibit the shortest duration of activation immediately after the acquisition session, as indicated by measurements taken 60 min post-session (Fig. 3). Post hoc analyses revealed that NT animals demonstrated heightened levels of Arc mRNA in ACC, LIM, ENT, CA1, and CPu relative to TWO-, THREE-, EIGHT-, and ELEVEN-day groups (Fig. 6) and in perirhinal (PERI), SUB, and CA3 relative to all but the TWO-day group. Additionally, TWO-day animals showed significantly 

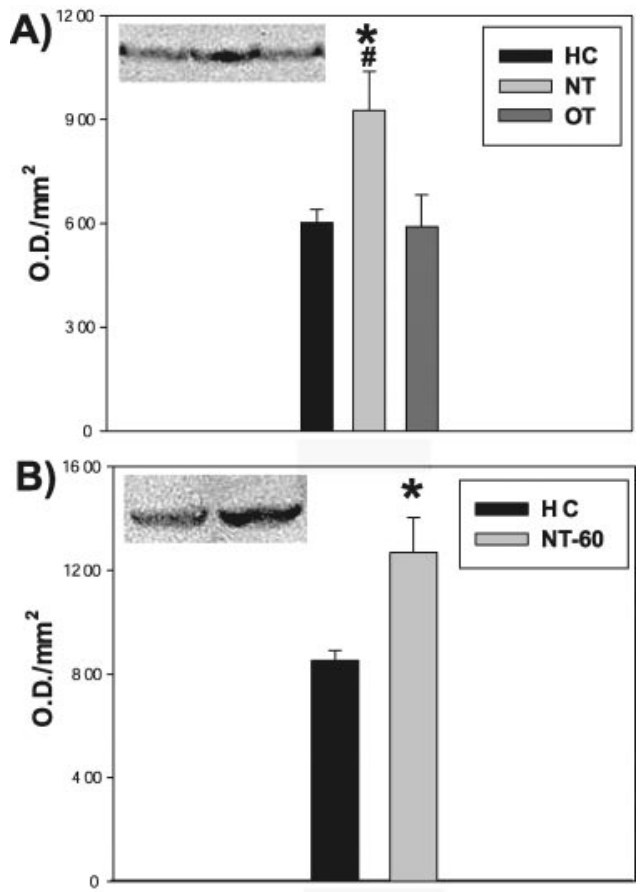

Figure 5. Detectable elevation of Arc protein measured exclusively in newly trained animals. $A$, Western blot from frontal cortex, comparing Arc protein levels in home-caged controls (HC), newly trained animals killed $30 \mathrm{~min}$ (NT) after the session, and overtrained animals (OT) killed 30 min after the session. $B$, Western blot from frontal cortex comparing the same $\mathrm{HC}$ animals with NT animals killed 60 min after the session (NT-60). Data are expressed as mean optical density per square millimeter (0.D. $\left./ \mathrm{mm}^{2}\right) \pm$ SEM. Post hoc analysis by Student-NewmanKeuls: significant increase versus $\mathrm{HC}^{*} p<0.05$, versus $0 \mathrm{~T}^{\#} p<0.05$.

more Arc mRNA induction in LIM, PERI, ENT, CA3, and insular cortex (INS) than the THREE-, EIGHT-, and ELEVEN-day groups, and in CA1 and SUB relative to the EIGHT- and ELEVEN-day groups (Fig. 6). The EIGHT- and ELEVEN-day groups did not differ from each other in any of the ROIs studied (Fig. 6).

\section{Correlation of Arc mRNA levels with individual animal performance}

It was shown previously that animals that were slower to acquire the lever-press task demonstrated higher levels of Arc mRNA relative to animals that acquired the task more rapidly (Kelly and Deadwyler, 2002). Therefore, analyses of individual measures of Arc mRNA levels and task performance were examined for possible covariation. Within the NT group $(n=13)$, analyses revealed that the number of presses made during the shaping phase, before the animal was able to perform the task independently, showed slight positive correlations with Arc mRNA levels in ENT $(r=0.558 ; p<0.05)$ and PERI $(r=0.570 ; p<0.05)$. Furthermore, levels in ACC, LIM, CA3, CA1, SUB, ENT, PERI, and CPu of NT animals correlated negatively with independent LPRs (Table 1, Fig. $8 A$ ). Conversely, there were no correlations between Arc mRNA levels and LPRs of the TWO-day $(n=8)$ or EIGHTday groups $(n=13)$ (Table 1 , Fig. $8 B)$. A related measure, total session length, was also examined for covariation with Arc levels in NT and PT animals. Total session length correlated positively with NT Arc mRNA levels in ACC, LIM, CA3, CA1, SUB, ENT, PERI, CPu, and PIR (Table 2) but did not show any correlation in PT animals $(n=12)$ (Table 2$)$.

\section{Discussion}

The current study extended previous findings (Kelly and Deadwyler, 2002) by showing that the acquisition of a lever-press response resulted in upregulation of both Arc mRNA and protein (Figs. 3, 5). Importantly, emulsion showed localization of Arc mRNA over cell bodies and revealed linear deposits extending away from these clusters, suggesting migration of Arc mRNA into dendritic processes (Fig. 4) (Link et al., 1995; Lyford et al., 1995). Furthermore, by measuring the time course of decay in Arc mRNA levels as well as the persistence of induction across days of training, the results indicated that various brain regions underlying the lever-press behavior appear to have different durations of activation (Figs. 3, 6). Given that the time course of Arc induction paralleled the early stages of behavioral change and that the message appeared to migrate into dendrites, it is likely that Arc is involved in the consolidation of new learning (Link et al., 1995; Lyford et al., 1995; Guzowski et al., 2000, 2001; Kelly and Deadwyler, 2002). However, the fact that the experience-dependent regulation of Arc mRNA varied across brain areas indicates that the rate of consolidation of the newly acquired behavior is not necessarily the same in all brain regions.

\section{Levels of Arc induction decline with overtraining}

Although overtrained animals demonstrated upregulation of Arc mRNA above home-caged levels, newly trained animals exhibited even higher levels of induction in many regions of interest (Fig. 3), including hippocampus as well as parahippocampal and frontal cortical areas. Furthermore, newly trained animals showed significantly higher levels of Arc protein relative to home caged and overtrained animals in frontal cortex, with no difference between the latter groups (Fig. 5). The apparent dissociation between Arc mRNA expression and protein levels in overtrained animals (Figs. 3, 5) may reflect multiple stages of regulation of this gene (i.e., transcription and translation).

Because the current study was not designed to elucidate the specific role of a given region in the acquisition of this behavior, it is not possible to rule out the prospect that the observed increases in Arc expression were a generalized response to any learning experience. Although this simple lever-press task is regarded as hippocampal independent, previous research has shown that the hippocampus encodes task-specific information, even when that information is not required on a particular trial (Deadwyler et al., 1985; Foster et al., 1987). Furthermore, the fact that there were tight correlations between behavioral measures and Arc expression in newly trained animals (see below) suggests that there is task specificity to the increases noted in Arc expression, supporting the hypothesis that Arc is involved in the synaptic mechanisms that result in the consolidation of newly acquired behaviors.

\section{Rate of decay of Arc mRNA levels varies across brain regions} By 60 min after the session, Arc mRNA levels in newly trained animals returned to baseline in CA3, CA1, central nucleus of the amygdala, and perirhinal, entorhinal, and insular cortices but remained elevated in infra/prelimbic, somatosensory, anterior cingulate, and piriform cortices as well as in the subiculum and caudate-putamen (Fig. 3). This would suggest that the period of Arc mRNA induction was extended, or that the rate of its subsequent degradation was less rapid, in some brain areas versus others. A similar differential involvement of brain structures over time was shown by Sif and colleagues (1991) using $\left[{ }^{14} \mathrm{C}\right] 2$ deoxyglucose metabolic mapping. They observed significant in- 


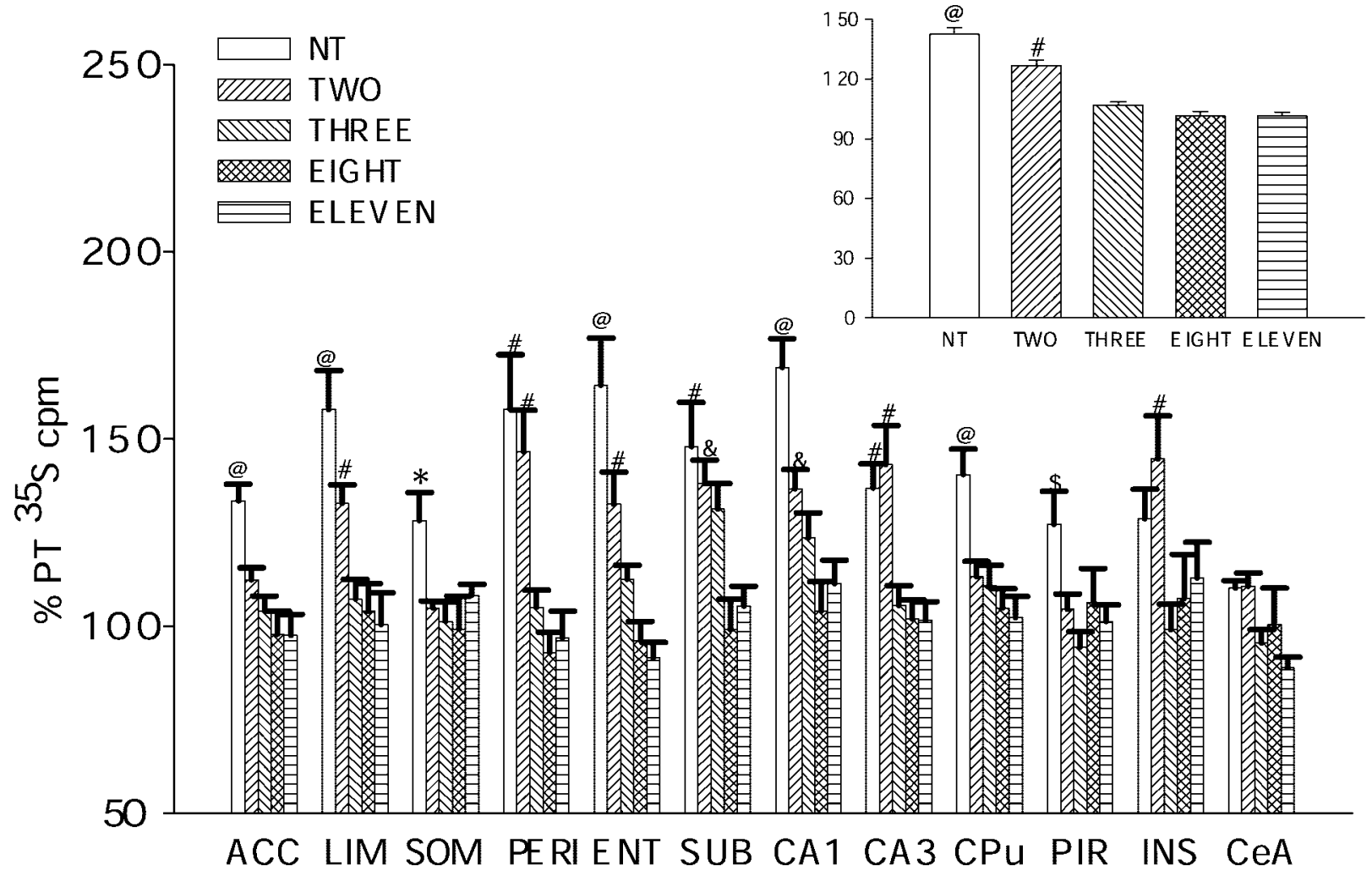

Figure 6. Persistence of Arc mRNA induction varies across different brain regions. Autoradiographic data $\left({ }^{35} \mathrm{~S} \mathrm{cmm}\right.$ ) for all regions of interest for newly trained (NT), TWO-, THREE-, EIGHT-, or ELEVEN-day groups $(n=8$ for each). Inset shows group means averaged across all areas. Data are expressed as a percentage of the pseudotrained (PT) group mean \pm SEM. ACC, Anterior cingulate cortex; LIM, infra/prelimbic cortex; SOM, somatosensory cortex; PERI, perirhinal cortex; ENT, entorhinal cortex; SUB, subiculum; CPu, caudate-putamen; PIR, piriform cortex; INS, insular cortex; CeA, central nucleus of the amygdala. Post hoc analysis by Student-Newman-Keuls: significant increase ${ }^{\circledR}$ versus TWO, THREE, EIGHT, and ELEVEN $p<0.05-p<0.001$; \# ${ }^{*}$ versus THREE, EIGHT, and ELEVEN $p<0.05-p<0.001$; ${ }^{*}$ versus THREE and EIGHT $p<0.05 ;{ }^{5}$ versus THREE and ELEVEN $p<0.05-0.01$; ${ }^{\&}$ versus EIGHT and ELEVEN $p<0.05-p<0.001$.

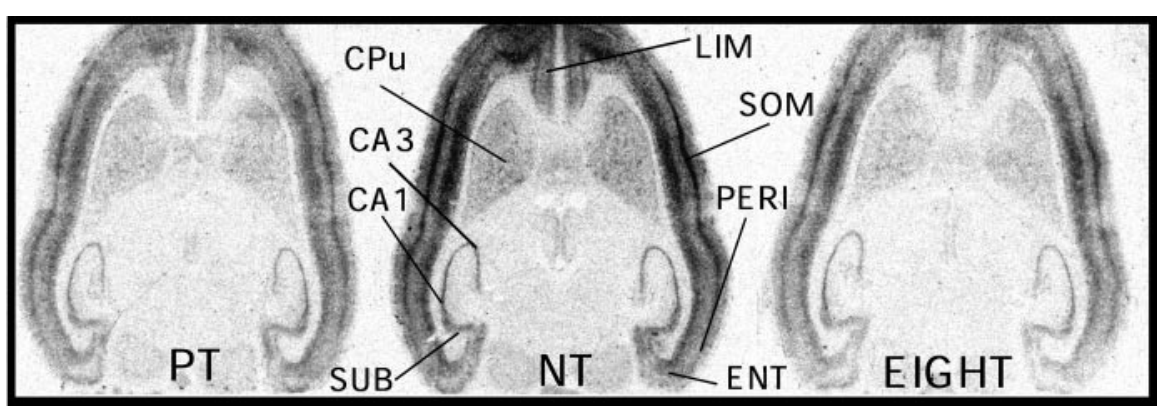

Figure 7. Heightened Arc mRNA levels in newly trained animals. Autoradiographic images of horizontal sections (DV 4.1$5.1 \mathrm{~mm}$ bregma) hybridized with ${ }^{35}$ S-labeled Arc antisense probes show labeling in pseudotrained (PT), newly trained (NT), and EIGHT-day animals of experiment 2. Brightness and contrast of images were heightened for clarity and definition.

(Hampson et al., 1999). The fact that Arc levels decrease first in hippocampal and parahippocampal areas but remain elevated in anterior cingulate cortex may reflect the flow of information along the anterior cingulate circuit, from hippocampal and parahippocampal regions to anterior cingulate. It remains to be determined whether the elevation in Arc levels noted 60 min post-session reflects a prolonged activation or subsequent reactivation of brain regions after the session.

\section{Persistence of Arc mRNA induction varies across brain regions}

Significant upregulation of Arc mRNA

creases in hippocampus and frontal, cingulate, and sensory motor cortices $15 \mathrm{~min}$ after lever-press training; however, at 220 min, significant activation was seen in frontal, cingulate, and piriform cortices as well as subiculum. It should be noted that Arc levels measured at $60 \mathrm{~min}$ were not simply a function of signal intensity noted $30 \mathrm{~min}$ after the session, because areas that initially had both the highest (somatosensory) and lowest (caudateputamen) levels of Arc mRNA demonstrated elevated levels 60 min after the session.

The differential Arc labeling across brain regions $60 \mathrm{~min}$ after the session may reflect the anatomical flow of information across brain structures, for example, information about reinforcing stimuli in anterior cingulate cortex (Freeman et al., 1996a,b; Parkinson et al., 2000) and spatial information in hippocampus persisted beyond the first day of training (Fig. 6), coinciding with the occurrence of spontaneous improvement on the lever-press task, which was noted between sessions 1, 2, and 3, but not thereafter (Fig. 2C). This suggests that Arc plays a role in post-session consolidation mechanisms responsible for spontaneous improvement (Mathis and Ungerer, 1999). The continued presence of significantly elevated Arc levels in animals trained for $2 \mathrm{~d}$ is a critical finding because it confirms that the effect noted in newly trained animals is not related strictly to experimenter intervention during the shaping procedure. Although increased Arc induction continued during the period of improving behavioral performance, the persistence of this induction differed across brain regions (Fig. 6). For example, marginal levels of Arc mRNA 
Table 1. Correlations of Arc mRNA levels and lever-pressing rates of individual newly trained (NT), TW0-day, and EIGHT-day animals

\begin{tabular}{|c|c|c|c|}
\hline Region & NT $(n=13)$ & TW0 $(n=8)$ & $\operatorname{EIGHT}(n=13)$ \\
\hline Anterior cingulate & -0.673 & -0.285 & 0.076 \\
\hline Infra/prelimbic & -0.604 & 0.240 & 0.178 \\
\hline Somatosensory & -0.453 & -0.131 & -0.076 \\
\hline Perirhinal & -0.659 & 0.328 & 0.021 \\
\hline Entorhinal $^{a}$ & -0.712 & 0.027 & 0.116 \\
\hline Subiculum & -0.626 & -0.158 & -0.199 \\
\hline$C A 1^{a}$ & -0.653 & -0.279 & 0.024 \\
\hline CA3 & -0.696 & -0.311 & 0.146 \\
\hline Caudate-putamen & -0.709 & -0.203 & -0.248 \\
\hline Piriform & -0.487 & -0.667 & -0.025 \\
\hline Insular & -0.258 & -0.149 & 0.016 \\
\hline Central nucleus of the amygdala & -0.146 & 0.398 & -0.029 \\
\hline
\end{tabular}

Correlations conducted by Pearson product moment correlation $(r)$, with statistical significance of $p<0.05-p<$ 0.01 indicated in bold.

${ }^{\text {a }}$ Regions plotted in Figure $8, A$ and $B$.

Table 2. Correlations of Arc mRNA levels and total session length of individual newly trained (NT) and pseudotrained (PT) animals

\begin{tabular}{llr}
\hline Region & NT $(n=13)$ & PT $(n=12)$ \\
\hline Anterior cingulate & $\mathbf{0 . 5 7 1}$ & 0.027 \\
Infra/prelimbic & $\mathbf{0 . 5 7 0}$ & 0.147 \\
Somatosensory & 0.529 & -0.270 \\
Perirhinal & $\mathbf{0 . 6 3 8}$ & -0.385 \\
Entorhinal & $\mathbf{0 . 7 0 3}$ & -0.224 \\
Subiculum & $\mathbf{0 . 6 3 8}$ & 0.046 \\
CA1 & $\mathbf{0 . 6 3 9}$ & -0.299 \\
CA3 & $\mathbf{0 . 6 0 9}$ & -0.090 \\
Caudate-putamen & $\mathbf{0 . 7 3 6}$ & -0.160 \\
Piriform & $\mathbf{0 . 6 1 6}$ & 0.204 \\
Insular & 0.343 & 0.071 \\
Central nucleus of the amygdala & 0.233 & -0.116
\end{tabular}

Correlations conducted by Pearson product moment correlation ( $r$, with statistical significance of $p<0.05-p<$ 0.01 indicated in bold.

were still present in CA1 and subiculum after the third training session, whereas Arc mRNA in areas such as somatosensory and anterior cingulate cortex were reduced to overtrained levels by the second day of training. This suggests that the involvement of the latter areas may be restricted to the initial stages of training, as indicated by enhanced Arc expression only on the day the lever-press response was initially acquired. Conversely, hippocampal and parahippocampal areas appear to process information regarding improvement across subsequent training sessions as well as that related to initial acquisition. It is interesting to note that, in general, brain areas that demonstrated the least persistence in Arc mRNA expression immediately after the session (e.g., hippocampal and parahippocampal regions) (Fig. 3) showed the most perseverance of induction across days of training (Fig. 6).

\section{Slower learners demonstrate higher} levels of Arc mRNA

The present study confirmed previous observations in which newly trained animals that were slower to acquire the lever-press task during the session exhibited higher levels of Arc mRNA (Fig. 8A) than animals that learned more quickly (Kelly and Deadwyler, 2002). This effect appears to be restricted to the day of acquisition (newly trained animals), because no significant correlations were noted for animals trained for 2 or $8 \mathrm{~d}$ (Fig. $8 \mathrm{~B}$ ). Given its association with the cytoskeleton (Lyford et al., 1995) and the postsynaptic density (Husi et al., 2000), higher levels of Arc mRNA in slower learners may reflect "overstabilized" synaptic architecture that is more resistant to the plastic changes that underlie processing of new information (van Rossum and Hanisch, 1999). In fact, in human embryonic kidney 293 cells, Arc has been shown to bind to PSD-95 (postsynaptic density95) and SAP-97 (Synapse-associated protein 97) (Chowdhury et al., 2002) and overexpression of Arc in organotypic hippocampal slice cultures has been shown to suppress AMPA receptor-mediated synaptic transmission (Verde et al., 2002). The present finding that Arc induction was associated with learning, but that slower learners demonstrated the highest levels of Arc, suggests there may be an optimal range of Arc expression for the synaptic modification associated with learning.

In conclusion, the present study demonstrated that newly trained animals induced higher levels of both Arc mRNA and Arc protein relative to home cage and overtrained animals. Importantly, heightened Arc mRNA induction across days coincided specifically with the occurrence of spontaneous improvement on the lever-press task, and behaviorally induced Arc mRNA appeared to migrate into dendrites. Taken together, the present results support the hypothesis that Arc plays an important role in synaptic processes during the establishment of new behaviors. Interestingly, the persistence of Arc mRNA levels, both immediately after the acquisition session as well as across days of training, varied across brain regions, suggesting a differential contribution
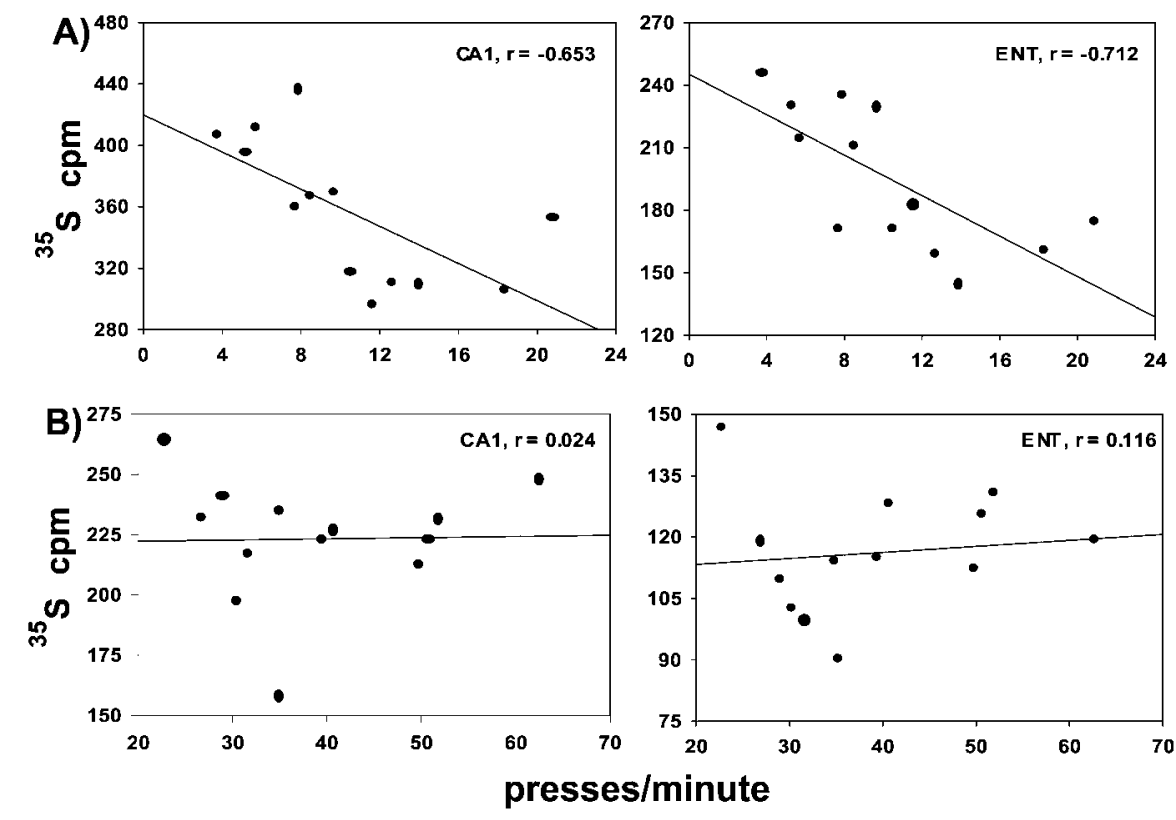

Figure 8. Animals that were slower to acquire the task had higher levels of Arc mRNA than faster learners. A, Representative correlations of lever-pressing rates and autoradiographic data ${ }^{35} \mathrm{~S} \mathrm{~cm}$ ) from $\mathrm{CA} 1$ and entorhinal cortex (ENT) show that Arc mRNA levels in NT animals ( $n=13$ ) correlated negatively with independent lever-pressing rates (CA1, $p<0.05$; ENT, $p<0.01$ ). $B$, There were no significant correlations between Arc mRNA levels and lever-pressing rates of EIGHT-day animals. Analyses by Pearson product moment correlation $(r)$. 
of various brain structures to the consolidation of the newly acquired behavior as it becomes firmly established.

\section{References}

Bailey CH, Kandel ER (1993) Structural changes accompanying memory storage. Annu Rev Physiol 55:397-426.

Bear MF (1997) How do memories leave their mark? Nature 385:481-482.

Chowdhury S, Petralia RS, Yang L, Okuno H, Worley PF (2002) Arc is a synaptic adaptor for SH3 and SH3-GK domain proteins. Soc Neurosci Abstr 28:746.13.

Deadwyler SA, West MO, Christian EP, Hampson RE, Foster TC (1985) Sequence-related changes in sensory-evoked potentials in the dentate gyrus: a mechanism for item-specific short-term information storage in the hippocampus. Behav Neural Biol 44:201-212.

DiAntonio A (2000) Neurobiology—translating activity into plasticity. Nature 405:1011-1012.

Foster TC, Christian EP, Hampson RE, Campbell KA, Deadwyler SA (1987) Sequential dependencies regulate sensory evoked responses of single units in the rat hippocampus. Brain Res 408:86-96.

Freeman Jr JH, Cuppernell C, Flannery K, Gabriel M (1996a) Contextspecific multi-site cingulate cortical, limbic thalamic, and hippocampal neuronal activity during concurrent discriminative approach and avoidance training in rabbits. J Neurosci 16:1538-1549.

Freeman Jr JH, Cuppernell C, Flannery K, Gabriel M (1996b) Limbic thalamic, cingulate cortical and hippocampal neuronal correlates of discriminative approach learning in rabbits. Behav Brain Res 80:123-136.

Freeman WM, Brebner K, Lynch WJ, Robertson DJ, Roberts DC, Vrana KE (2001) Cocaine-responsive gene expression changes in rat hippocampus. Neuroscience 108:371-380.

Frey U, Morris RG (1998) Synaptic tagging: implications for late maintenance of hippocampal long-term potentiation. Trends Neurosci 21:181-188.

Gall CM, Hess US, Lynch G (1998) Mapping brain networks engaged by, and changed by, learning. Neurobiol Learn Memory 70:14-36.

Guthrie K, Rayhanabad J, Kuhl D, Gall C (2000) Odors regulate Arc expression in neuronal ensembles engaged in odor processing. NeuroReport 11:1809-1813.

Guzowski JF, Lyford GL, Stevenson GD, Houston FP, McGaugh JL, Worley PF, Barnes CA (2000) Inhibition of activity-dependent Arc protein expression in the rat hippocampus impairs the maintenance of long-term potentiation and the consolidation of long-term memory. J Neurosci 20:3993-4001.

Guzowski JF, Setlow B, Wagner EK, McGaugh JL (2001) Experiencedependent gene expression in the rat hippocampus after spatial learning: a comparison of the immediate-early genes Arc, c-fos, and zif268. J Neurosci 21:5089-5098.

Hampson RE, Simeral JD, Deadwyler SA (1999) Distribution of spatial and nonspatial information in dorsal hippocampus. Nature 402:610-614

Husi H, Ward MA, Choudhary JS, Blackstock WP, Grant SG (2000) Proteomic analysis of NMDA receptor-adhesion protein signaling complexes. Nat Neurosci 3:661-669.

Kelly MP, Deadwyler SA (2002) Acquisition of a novel behavior induces higher levels of Arc mRNA than does overtrained performance. Neuroscience 110:617-626.

Laemmli UK (1970) Cleavage of structural proteins during the assembly of the head of bacteriophage T4. Nature 227:680-685.

Link W, Konietzko U, Kauselmann G, Krug M, Schwanke B, Frey U, Kuhl D (1995) Somatodendritic expression of an immediate early gene is regulated by synaptic activity. Proc Natl Acad Sci USA 92:5734-5738.

Lyford GL, Yamagata K, Kaufmann WE, Barnes CA, Sanders LK, Copeland NG, Gilbert DJ, Jenkins NA, Lanahan AA, Worley PF (1995) Arc, a growth factor and activity-regulated gene, encodes a novel cytoskeletonassociated protein that is enriched in neuronal dendrites. Neuron 14:433-445.

Mathis C, Ungerer A (1999) The retention deficit induced by (RS)-alphamethyl-4-carboxyphenylglycine in a lever-press learning task is blocked by selective agonists of either group I or group II metabotropic glutamate receptors. Exp Brain Res 129:147-155.

Montag-Sallaz M, Welzl H, Kuhl D, Montag D, Schachner M (1999) Novelty-induced increased expression of immediate-early genes c-fos and $\arg 3.1$ in the mouse brain. J Neurobiol 38:234-246.

Moser MB (1999) Making more synapses: a way to store information? Cell Mol Life Sci 55:593-600.

Parkinson JA, Willoughby PJ, Robbins TW, Everitt BJ (2000) Disconnection of the anterior cingulate cortex and nucleus accumbens core impairs Pavlovian approach behavior: further evidence for limbic cortical-ventral striatopallidal systems. Behav Neurosci 114:42-63.

Paxinos G, Watson C (1997) The rat brain in stereotaxic coordinates, Ed 3. San Diego: Academic Press.

Sif J, Messier C, Meunier M, Bontempi B, Calas A, Destrade C (1991) Timedependent sequential increases in $\left[{ }^{14} \mathrm{C}\right] 2$-deoxyglucose uptake in subcortical and cortical structures during memory consolidation of an operant training in mice. Behav Neural Biol 56:43-61.

Smith PK, Krohn RI, Hermanson GT, Mallia AK, Gartner FH, Provenzano MD, Fujimoto EK, Goeke NM, Olson BJ, Klenk DC (1985) Measurement of protein using bicinchoninic acid [Erratum (1987) 163:279]. Anal Biochem 150:76-85.

Steward O, Worley PF (2001) Selective targeting of newly synthesized Arc mRNA to active synapses requires NMDA receptor activation. Neuron 30:227-240.

Steward O, Wallace CS, Lyford GL, Worley PF (1998) Synaptic activation causes the mRNA for the IEG Arc to localize selectively near activated postsynaptic sites on dendrites. Neuron 21:741-751.

Stork O, Welzl H (1999) Memory formation and the regulation of gene expression. Cell Mol Life Sci 55:575-592.

van Rossum D, Hanisch UK (1999) Cytoskeletal dynamics in dendritic spines: direct modulation by glutamate receptors? Trends Neurosci 22:290-295.

Verde EMR, Worley PF, Malinow R, Cline HT (2002) The role of Arc in synaptic function in rat hippocampus. Soc Neurosci Abstr 28:839.5.

Yin Y, Edelman GM, Vanderklish PW (2002) The brain-derived neurotrophic factor enhances synthesis of Arc in synaptoneurosomes. Proc Natl Acad Sci USA 99:2368-2373. 